\title{
Su alcune disposizioni elettrodiche interessanti processi elettrocinetici in terreni saturi d'acqua
}

\author{
1. CREA
}

Studiamo il fenomeno elettrogeosmotico, nel caso stazionario di un campione di terreno saturo d'acqua in cui si facecia fluire corrente elettrica mediante due elettrodi indefiniti, (ilindrici non coassiali ( $r$. fig. 1a), o cilindrico l'anodo, piano il catodo e parallelo alle generatrici anodiche (r. fig. $1 b$ ).

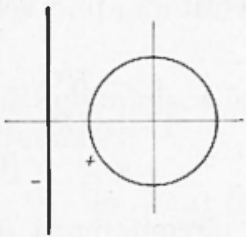

Fig. 1a

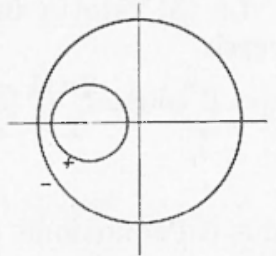

Fig. $1 b$
Supporremo che all'interno dell'anodo si eserciti artificialmente una pressione idraulicar $H_{A}$ magriore o nguale della pressione esistente al catodo $\left(H_{K}\right)$. Sia anodo che catorlo si penseranno permeabili all'acqua, per esempio forellati.

Useremo i simboli seguenti:

$\bar{q}=$ vettore di flusso idlaulico;

$\bar{j}=$ vettore densità di corrente elettrica

$H=$ pressione idraulica nel punto generico del campione;

$V=-$ potenziale elettrico nel punto generico del rampione;

$H_{A}=$ pressione idraulica anodica

$H_{K}=$ pressione idraulica catodica

$V_{A}=$ potenziale anodico

$V_{K}-$ potenziale catodico $\sigma=$ conduttività elettrica del terreno

$I=$ corrente elettrica totale

$\Gamma=$ resistenza elettrica:

$k_{i}=$ coefficiente di permeabilità idraulica (D)arcy)

$k_{c}=$ coefficiente elettroosmotico

$\mu=k_{e} / \sigma=$ coefficiente mediamente invariantivo.

\section{$\$ 1$.}

L'equazione fondamentale del fenomeno (che congloba le legge di Darey ed Helmholz), è

$$
\bar{q}=-l i \text { grad } H+\mu \bar{j}
$$

c Ie condizioni di continuità

$$
\text { di } v \bar{q}=0, \text { di } v \bar{j}=0 \text {. }
$$

Inoltre, detto $r(x, y)$ il potenziale elettrico, si avrà anche

$$
\bar{j}=-\sigma \operatorname{grad} V
$$

Applicando l'operatore divergenza alla [1] e tenendo conto delle $\left[\mathbf{1}^{\prime}\right]$, si ottiene subito

$$
\text { di } v(\operatorname{mlad} H)=\frac{\partial^{2} H}{\partial x^{2}}+\frac{\partial^{2} H}{\partial y^{2}}=0
$$

e dalla $\left[1^{\prime \prime}\right]$, applicando ancora l'operatore divergenza e tenendo conto della seconda delle $\left[1^{\prime}\right]$

$\operatorname{di} v \bar{j}=-\sigma \operatorname{di} v\left(\operatorname{grad} V^{V}\right)=-\sigma\left(\frac{\lambda^{2} V}{\partial v^{2}}+\frac{\partial^{2} V}{\partial y^{2}}\right)=0$. 
Alle [2] e [3] andranno agogiunte le rondizioni al contorno, come segue:

per la [2]:

$$
H(x, y)=H_{A} \text { sull'anodo, }
$$

per lat [3]

$$
H(x, y)=H_{K} \text { sul catodo, }
$$

$$
\begin{aligned}
& Y(x, y)=V_{A} \text { sull'anodo, } \\
& V(x, y)=V_{K} \text { sul catodo. }
\end{aligned}
$$

Il problema, pertanto, è identico sia per la pressione idrostatica che per il potenziale elettrico: trovare una funzione armonica $\varphi(x, y)$ (che può essere la pressione 0 il potenziale elettrico) che su anodo e catodo assuma valori costanti prefissati.

\section{$\S 2$.}

Allo scopo di risolvere il problema di Dirichlet ora posto, introduciamo i due piani complessi $(z=x+i y)$ e $w=u+i v)$, e tra essi poniamo la corrispondenza subordinata dalla funzione complessa

$$
w=\frac{z-a}{a z-1}
$$

(a costante reale $>1$ ), analitica nel piano $z$ salvo nel punto $z=\frac{1}{a}$ in cui essa ha una singolarità polare del primo ordine.

Come è ben noto dalla teoria delle funzioni di una variabile complessa, la corrispondenza cosi posta fra i piani $(z)$ e $(w)$ è generalmente conforme, nel senso che vengono conservati gli angoli formati fra loro da due arehi di eurva uscenti da un punto del piano $(z)$, nel passaggio al punto e alle curve corrispondenti del piano $(w)$; fanno eccezione le singolarità della funzione, e i punti in cui si annulla la derivata della funzione.

ì altresì noto, dalla teoria delle funzioni armoniche, che una trasformazione conforme nel piano muta funzioni armoniche in funzioni armoniche.

Studiamo ora brevemente la corrispondenza definita dalla [5], mettendone in evidenza alcune proprietà fondamentali.
Consideriamo dapprima una circonferenza nel piano $w$, di centro nel punto $w=0$ e raggio arbitrurio $R$; essa arrà equazione

$$
|w|^{2}=R^{2}
$$

ove $|w|$ ¿̀ il modulo di $w$; indicando con sopralineature i coniugati dei numeri complessi, potremo scrivere la precedente equazione:

$$
\begin{gathered}
|w|^{2}=w \cdot w=\frac{z-a}{a z-1} \cdot \frac{z-a}{a z-1}= \\
-\frac{|z|^{2}-2 a R(z)+a^{2}}{a^{2}|z|^{2}-2 a R(z)+1} .
\end{gathered}
$$

Ma $z^{2}=x^{2}+y^{2}, R(z)=x$, quindi l'equazione precedente si scrive, dopo ovvie semplificazioni:

$$
\frac{(x-a)^{2}+y^{2}}{(a x-1)^{2}+a^{2} y^{2}}=R^{2} .
$$

La [3] ridotta in forma intera, può scriversi:

$$
x^{2}+y^{2}-\frac{2 a\left(1-R^{2}\right)}{1-a^{2} R^{2}} x+\frac{a^{2}-R^{2}}{1-a^{2} R^{2}}=0
$$

che è l'equazione di una circonforenza del piano $(z=x+i y)$ di centro $\left(\frac{a\left(1-R^{2}\right)}{1-u^{9} R^{\prime}}, 0\right)$, e di raggio $\left(\frac{R\left(1-a^{2}\right)}{1-R^{2} a^{2}}\right)$ (si noti che ciò comporta, essendo $\left.a>1, R>\frac{1}{a}\right)$, che è la curva corrispondente, nel piano $(z)$ alla generica circonferenza con centro nell'origine del piano $(w)$. Si noti poi che alla circonferenza di raggio $R=1$ e centro nell'origine del piano $(w)$ corrisponde la circonferenza di raggio 1 e centro nell'origine del piano $z$, qualunque sia $a$.

Alla circonferenza del piano (w) con centro nell'origine e raggio $h=\frac{1}{a}$ corrisponde una circonferenza di ragrio infinito, e con centro all'infinito nella direzione dell'asse delle $x$ : cioè la retta di equazione (come si ricava sempre dalla [6])

$$
x=\frac{a^{2}+1}{2 a}
$$


parallela all'asse $y$ e a distanza $\frac{a^{2}+1}{\sigma a}>1$ da esso.

Sia ora una retta per l'origine del piano $w$; detto $w_{0}=u_{0}+i v_{0}$ un suo punto, l'equazione di tale retta puó seriversi:

$$
w=t w_{n}
$$

essendo $t$ un parametro reale. La curva corrispondente nel piano $(z)$ soddisfa alla relazione

$$
\frac{z-a}{a z-1}=w_{0} t
$$

da cui, ponendo $z=x+i y$ e separando il reale clall'immaginario

$$
\begin{gathered}
\frac{(x-a)(a x-1)+a y^{2}}{(a x-1)^{2}+a^{2} y^{2}}=u_{0} t, \\
\frac{\left(a^{2}-1\right) y}{(a x-1)^{2}+a^{2} y^{2}}=v_{0} t .
\end{gathered}
$$

Eliminando $t$ fra le [9] e posto $\frac{\ddot{w}_{0}}{v_{0}}=\lambda$ otteniamo:

$$
\frac{(x-a)(a x-1)+a y^{2}}{\left(a^{2}-1\right) y}=\lambda
$$

riocis

$$
x^{2}+y^{2}-\frac{a^{2}+1}{a} x-\lambda \frac{a^{2}-1}{a} y+1=0
$$

che è l'equazione di una circonferenza di centro $\left(\frac{a^{2}+1}{2 a}, \lambda \frac{a^{2}-1}{2 a}\right)$ e di raggio $\left(\mid 1+\lambda^{2} \frac{a^{2}-1}{2 a}\right)$, passante, qualunque sia $\lambda$, per i punti $(a, o)$ e $\left(\frac{1}{a}\right.$, o : inoltre, al variare di $\lambda$, i centri di tali circonferenze hamno ascissa costante, quindi sono situati su una retta che coincide con quella di equazione [7].

Le rirconferenze di equazione [6] e [10] sono mutuamente ortogonali, come assicura il carattere conforme della corrispondenza, e come è del resto facile verificare direttamente; per ogni punto del piano $(z=x+i y)$ lie passa una ed una sola di ciascuma famiglia, eceetto che per i punti, singrolari per la corrispondenza, $(a, o),\left(\frac{1}{a}, o\right)$ per eui non passa nessuna delle circonferenze [6] e passimo tutte le [10].

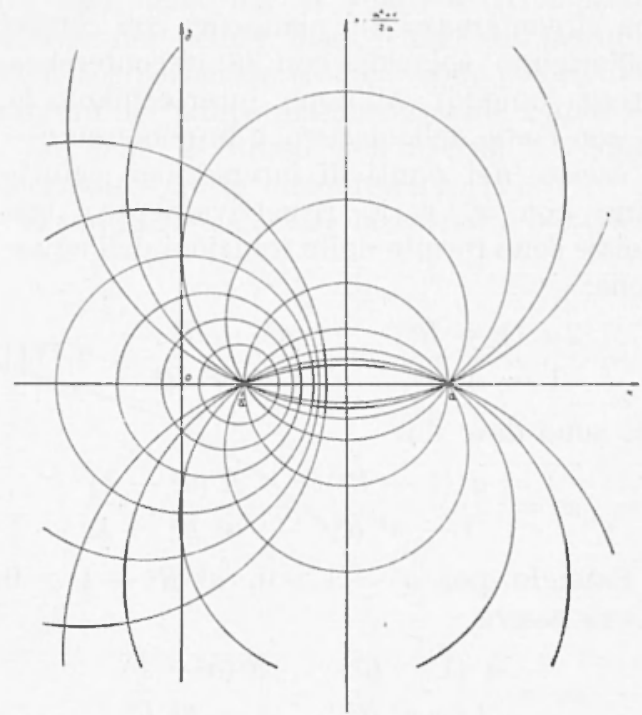

Fig. 2

La figr. 2 mostra l'andamento dei due sistemi di circonferenze [6] e [10].

\section{$\S 3$.}

Con l'ausilio della corrispondenza studiata nel paragrafo precedente, mostreremo nel presente paragrafo come sia possibile trasformare il dominio compreso fra i nostri elettrodi, pensato appartenente al piano $(z)$, in una corona circolare nol piano $(w)$. Assumiamo l'asse delle ascisse passante per i centri delle circonferenze sezioni degli elettrodi e consideriamo come coordinate di un punto del piano $(z)$ i rapporti fra le coordinate eflettive e il raggio della circonferenza catodica esterna (il che, orviamente, corrisponde a scegliere tale raggio come unita di misura), il che comporta che la circonferenza esterna avià, nel nuovo sistema di coordinate (" coordinate ridotte") maggio unitario. Siano $x_{1}$ e $x_{2}\left(-1<x_{1}<x_{2}<1\right)$ le coordinate ridotte delle intersezioni della circonferenza interma coll'asse delle ascisse.

Si è già visto (v' $\$ 2$ ) che la circonferenza di ragrio unitario nel piano $(z)$ corrisponde alla circonferenza unitaria nel piano $(w)$; per conseguire il nostro scopo, basterà far vedere 
che ì possibile specializzare nella [6] a ed $R$, in modo che la circonferenza che ne risulta (corrispondente, come si è visto, a una circonferenza del piano (w) con centro nell'origine) roincida con la circonferenza interna (anodo). All'uopo, intersechiamo la [6] con l'asse delle ascisse, e imponiamo che le ascisse dei punti di intersezione roincidano con $x_{1}$ e $x_{2}$ rispettivamente: tali ascisse sono fornite dalle soluzioni dell'equazione:

$x^{2}-\frac{2 a\left(1-R^{2}\right)}{1-a^{2} R^{2}} x+\frac{a^{2}-R^{2}}{1-a^{2} R^{2}}=0$

che sono date da:

$$
x=\frac{a\left(1-R^{2}\right)}{1-a^{2} R^{2}} \pm \frac{R\left(a^{2}-1\right)}{a^{2} R^{2}-1}
$$

Essendo poi $a^{2}-1>0, a^{2} R^{2}-1>0$ dovià esssere

$$
\begin{aligned}
& x_{1}=\frac{a\left(1-R^{2}\right)}{1-a^{2} R^{2}}-\frac{R\left(a^{2}-1\right)}{1-a^{2} R^{2}}, \\
& x_{9}=\frac{a\left(1-R^{2}\right)}{1-a^{2} R^{2}}+\frac{R\left(a^{2}-1\right)}{a^{2} R^{2}-1} .
\end{aligned}
$$

Gon qualche alcolo, è facile verlere rhe le [12] comportano le due equazioni:

$\left(x_{2}+x_{1}\right) a^{2}-2\left(1+x_{1} x_{2}\right) a+\left(x_{2}+x_{1}\right)=0$ $\left(x_{2}-x_{1}\right) R^{2}-2\left(1-x_{1} x_{2}\right) R+\left(x_{2}-x_{1}\right)=0$

che ammettono le soluzioni

[12']

$$
\begin{aligned}
& a=\frac{1+x_{1} x_{2} \pm 1\left(\overline{\left(1-x_{1}{ }^{2}\right)\left(1-x_{2}{ }^{2}\right)}\right.}{x_{1}+x_{2}}, \\
& R=\frac{1-x_{1} x_{2} \pm \sqrt{\left(1-x_{1}{ }^{2}\right)\left(1-x_{2}{ }^{2}\right)}}{x_{2}-x_{1}}
\end{aligned}
$$

nelle quali vammo assunti segni roneordi. Poichè deve essere, per ipotesi, $a>1$, e le due soluzioni della prima delle $\left[12^{\prime}\right]$ sono positive e una inversa dell'altra, occorrerà scegliere nelle [13] il segno positivo, per cui è

$$
\begin{gathered}
a=\frac{1+x_{1} x_{2}+\sqrt{\left(1-x_{1}{ }^{2}\right)}\left(1-x_{2}{ }^{2}\right)}{x_{1}+x_{2}}, \\
R=\frac{1-x_{1} x_{2}+\sqrt{\left(1-x_{1}{ }^{2}\right)\left(1-x_{2}{ }^{2}\right)}}{x_{2}-x_{1}}
\end{gathered}
$$

e risulta anche $R>1$ (anche le soluzioni della seconda delle $\left[12^{\prime}\right]$ sono fra loro reciproche).
Pertanto l'assunto è dimostrato: notiamo esplicitamente che alla circonferenza interna nel piano $(z)$ rorrisponde la cireonferenza esterna nel piano (w).

\section{$\S 4$.}

Risolvianno oral il problema di Dirichlet posto nel \$ 1 . A questo scopo trasformiamo il dominio (ompreso fra i due elettrodi secondo le modalità del $\$ 3$, risolviamo il problema di Dirichlet per il dominio trasformato (corona circolare) e invertiamo lat trasformazione. Per quanto detto alla fine del $\S 3$, occorrerì consiclerare, nel piano (w), l'anodo esterno al catodo (v. fig. 3).
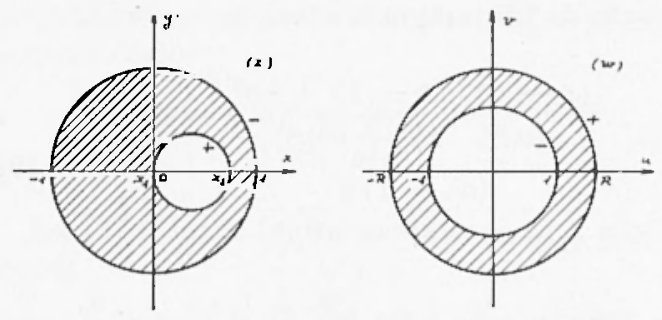

Fig. 3

Orviamente, per ragioni di simmetria, e per cose note, la funzione che risolse il nostro problema per la rorona circolare è del tipo:

$$
\varphi(r)=A \lg r+B
$$

con $r$ distanza del punto generico dall'origine, e $A$ e $B$ costanti da determinare con l'ausilio delle condizioni ai limiti.

Per la pressione irlostatica arremo

$$
H(R)=A \lg R+\mathrm{B}=H_{-t} \text { (sullanodo) }
$$$$
H(1)=B=H_{K}
$$

e quindi

$$
H(r)=\frac{H_{A}-H_{K}}{\lg R} \lg r+H_{K}
$$

e, con procedimento analogo per il potenziale elettrico $r(r)$,

$$
V^{*}(r)=\frac{V_{A}-V_{K}}{\lg R} \lg r+V_{\kappa}
$$

Tenendo conto rhe ̀े

$$
r=|w|=\sqrt{\frac{(x-a)^{2}+y^{2}}{(a x-1)^{2}+a^{2} y^{2}}}
$$


(per la [5]), otteniamo, nel piano $(Z=x+i y)$ $H(x, y)=\frac{H_{A}-H_{K}}{2 \lg R} \lg \frac{(x-a)^{2}+y^{2}}{(a x-1)^{2}+a^{2} y^{2}}+H_{K}$ $V(x, y)=\frac{V_{A}-V_{K^{K}}}{2 \lg R} \lg \frac{(x-a)^{2}+y^{2}}{(a x-1)^{2}+a^{2} y^{2}}+V_{K}$

che risolvono il nostro problema. Natural-
Come è facile vedere, e come ì fisieamente orvio, tale flusso catodico hat un amdamento del tipo della fig. 4, simmetrico rispetto all'asse dei centri degli elettrodi,massimo dove le superfici elettrodiche sono più vicine, minimo nel punto diametralmente opposto.

Le linee di flusso mellinterno dei due elettrodi, somo le cireonferenze [10].

È poi facile calcolare lia conrente alet rica

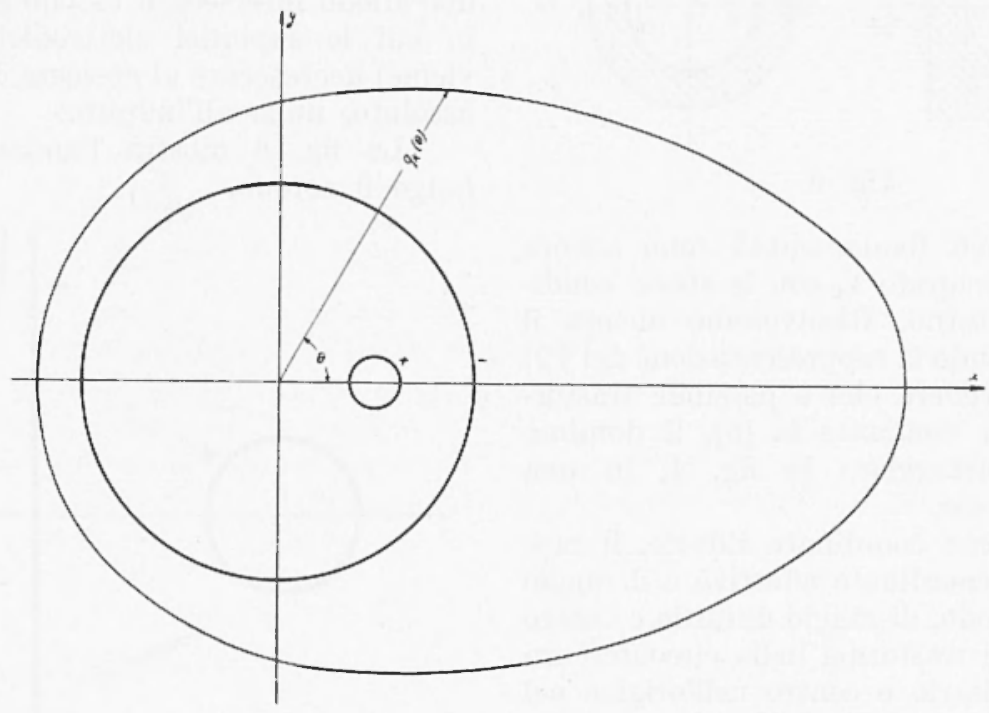

Fig. 4

mente, $(a)$ el $(R)$ lammo i ralori forniti dalle $\left[13^{\prime}\right]$.

Dalle [14], passando in coordinate polari nel piano $(z)$, con polo l'origine, otteniamo $H(\varrho, 0)=$

$=\frac{H_{A}-H_{K}}{2 \lg R} \lg \frac{\varrho^{2}-2 a \varrho \cos \theta+a^{2}}{a^{2} \theta^{2}-2 a \varrho \cos \theta+1}+H_{K}$ $\Gamma(\varrho, \theta)=$

$=\frac{V_{A}-V_{K}}{2 \lg R} \lg \frac{\varrho^{2}-2 a \varrho \cos \vartheta+a^{2}}{a^{2} \varrho^{2}-2 a \varrho \cos \vartheta+1}+Y_{K}$

da cui è facile risalire al flusso idrico catodico, mediante la [1]: infatti

$$
\begin{aligned}
& \Psi_{k}(\theta)=-k\left(\frac{\partial H}{\partial \varrho}\right)_{\varrho=1}-k_{c}\left(\frac{\partial V}{\partial \varrho}\right)_{\varrho=1}= \\
& =\frac{a^{2}-1}{\lg R} \frac{k\left(H_{A}-H_{K}\right)+k_{c}\left(V_{A}-V_{k}\right)}{a^{2}-2 a \cos \vartheta+1} .
\end{aligned}
$$

totale fluente fra gli elettrodi: basta ricorlare che, per la $\left[1^{\prime \prime}\right]$, è

c quindi

$$
\begin{gathered}
j_{k}=-\sigma\left(\frac{\partial V}{\partial g}\right)_{\varrho=1}= \\
=\frac{\sigma\left(a^{2}-1\right)}{1 g R} \frac{V_{A}-V_{k}}{a^{2}-2 a \cos \theta+1}
\end{gathered}
$$

$$
\begin{gathered}
\mathrm{I}=\int_{(k)} j_{k} d \cdot s= \\
=\frac{\sigma\left(a^{2}-1\right)\left(T_{A}-V_{K}\right) \int_{0}^{2 \pi} \frac{d \theta}{a^{2}-2 a \cos \theta+1}=}{=\frac{2 \pi \sigma\left(V_{A}-V_{K}\right)}{\lg R}}
\end{gathered}
$$

da cui la resistenza elettrica del dispositivo

$$
\Gamma=\frac{V_{A}-V_{K}}{I}=\frac{\lg R}{2 \pi \sigma} .
$$




\section{$\S$ э.}

Studiamo ora il caso in cui ad un anodo cilindrico indefinito, parallelo alle generatrici dell'anodo (r. fig. 5).
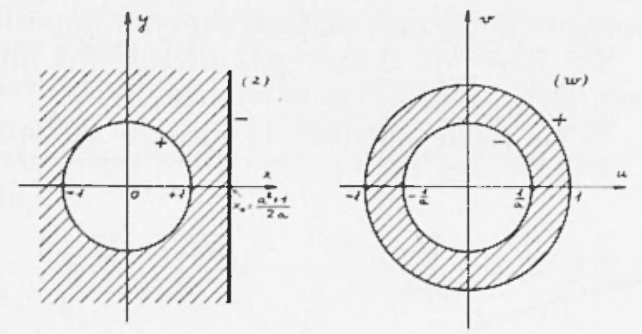

Fig. 5

Ise equazioni fondamentali sono ancora quelle del paragrafo 1 , con le stesse condizioni al contorno. Risolveremo ancora il problema usando la rappresentazione del § 2: basteri far vedere che è possibile trusformare, ancora mediante la [5], il dominio illimitato trattaggiato in fig. 4, in una corona circolare.

Usando come coordinate ridotte, il rapporto fra le coordinate effettive e il raggio anodico, l'anodo, di raggrio unitario e centro nell'origine si trasforma nella circonferenza di raggio unitario e centro nell'origine nel piano (w); per climostrare l'asserto, basterà far vedere detta $x_{0}$ la distanza ridotta del ratodo dell'origine, che è possibile determinare $a$ in modo che la retta [7] coincida col catodo; il che ovviamente comporta

$$
\frac{a^{2}+1}{2 a}=x_{n}
$$

rioè $a=x_{0}+1 x_{0}^{2}-1$ (dovendo essere $a>1)$. Con tale valore ali $a$, al catodo corrisponde nel piano (w) la rirconferenza di centro nell'origine e raggio $\frac{1}{a}<1$, interna alla circonferenza trasformata dell'anorlo.

Allora, con procedimento perfettamente analogo a quello del $\$ 4$, si giunge alle relazioni

$H(x, y)=\frac{H_{A}-H_{h}}{2 \lg a} \lg \frac{(x-a)^{2}+y^{2}}{(a x-1)^{2}+a^{2} y^{2}}+H_{A}$
$Y(x, y)=\frac{V_{A} T_{K} \lg \frac{(x-a)^{2}+y^{2}}{2 \lg a}+V_{A}}{(a x-1)^{2}+a^{2} y^{2}}+V_{A}$
Ini conseguenza, per il flusso catodico, si la

$$
\begin{aligned}
& q_{k}(y)=-k\left(\frac{\partial H}{\partial x}\right)_{x=x_{0}}-k_{e}\left(\frac{\partial V}{\partial x}\right)_{x=x_{0}}= \\
& \frac{4 a\left(a^{2}-1\right)}{\lg a} \frac{k\left(H_{A}-H_{K}\right)+K_{e}\left(V_{A}-V_{k}\right)}{\left(a^{2}-1\right)^{2}+4 a^{2} y^{2}}
\end{aligned}
$$

che è massima per $^{2} y-0$ (cioè nel punto in cui la normale al catodo per il centro dell'anodo interseca il catodo stesso; punto in cui le superfici elettrodiche sono più vicine) decrescente al crescere di $y$ in valore assoluto, nulla all'infinito.

La fig. 6 mostra l'andamento di $q_{k}$ lungo il catodo.

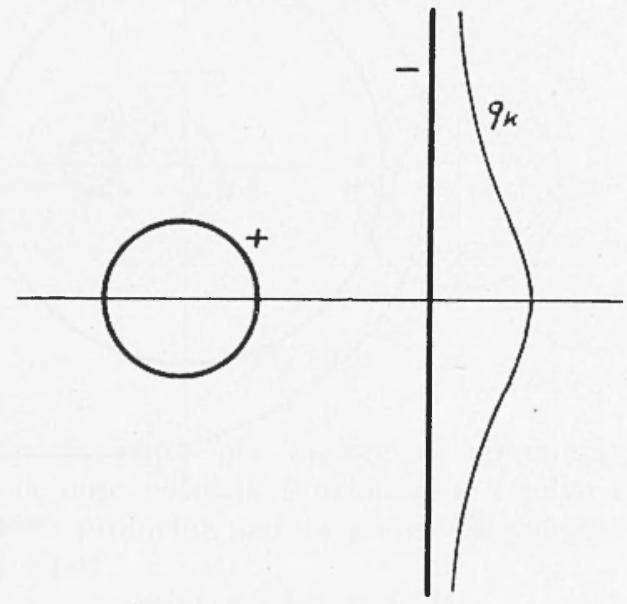

Fig. 6

Anche in tal caso è immediato il calcolo della corrente totale $I$ a della resistenza $I$.

Semple tenendo presente la (1"), abbiamo

$$
\begin{gathered}
j_{k}=-\sigma\left(\frac{\partial V}{\partial x}\right)_{x=x_{o}}= \\
=\frac{\sigma\left(V_{.1}-V_{K}\right)}{\lg a} \frac{1 a\left(a^{2}-1\right)}{\left(a^{2}-1\right)^{2}+4 a^{2} y^{2}}
\end{gathered}
$$

e quindi

$$
\begin{aligned}
& I=\int_{(\dot{k})} j_{k} d s= \\
& =\frac{4 a \sigma\left(a^{2}-1\right)}{\lg a}\left(V_{-1}^{(\dot{k})}+V_{-\infty}^{+\infty} \int_{-\infty}^{\infty} \frac{d y}{\left(a^{2}-1\right)^{2}+4 a^{2} y^{2}}=\right. \\
& =\frac{2 \pi \sigma\left(V_{A}-T_{K}\right)}{\lg a} \\
& \text { e } \quad \Gamma=\frac{V_{\mathrm{A}}-V_{K}}{I}=\frac{\lg a}{2 \pi \sigma} \text {. }
\end{aligned}
$$


CONClusione.

Le disposizioni elettrodiche qui studiate per la prima volta riguardano strutture di elettrodi cilindrici ad assi paralleli di cui uno è incluso totalmente nell'altro, cilindri coassiali, eccentrici, elettrodi esterni l'un l'altro, piano-cilindrici, ece.

Queste disposizioni polari possono ricor- rere frequentemente nella prospezione geoelettrica utilizzante quali elettrodi fori di sonda tubati anche parzialmente, ricorrono spesso nella elettroceotecniea in processi elettrosmotici bifilari introdotti dalla senola lussa (Netushil-Tomizó), sono dimostrative per quanto riguarda le caratteristiche di direzionabilità del nuovo processo dielettroiniezioni di leganti chimici. 